\footnotetext{
${ }^{7}$ L. Koester, Z. Phys. 182, 328 (1965), and $\underline{198,} 187$ (1967).

${ }^{8}$ L. Koester and W. Nistler, Z. Phys. A272, 189 (1975).

${ }^{9}$ A. W. Overhauser, R. Colella, and S. A. Werner, Phys. Rev. Lett. 34, 1472 (1975).

${ }^{10}$ N. Nücker, Z. Angew. Phys. 27, 33 (1969).

${ }^{11}$ L. D. Lucas, C. R. Acad. Sci., Ser. C 268, 1081 (1969).

${ }^{12}$ N. Nucker, Z. Phys. 227, 152 (1969).

${ }^{13}$ E. R. Cohen and B. N. Taylor, J. Phys. Chem. Ref. Data 2, 663 (1973).

${ }^{14}$ R. Scherm, Nuckleonik $\underline{12}, 4$ (1968).
}

\author{
${ }^{15} \mathrm{H}$. Glättli, private communication . \\ ${ }^{16}$ P. Roubeau, A. Abragam, G. L. Bacchella, H. Glät- \\ tli, A. Malinovski, P. Meriel, J. Piesveaux, and \\ M. Pinot, Phys. Rev. Lett. 33, 102 (1974). \\ ${ }^{17}$ V. E. Krohn and G. E. Ringo, Phys. Rev. D $\underline{8}, 1305$ \\ (1973). \\ ${ }^{18}$ E. Melkonian, B. M. Rustad, and W. W. Havens, Jr., \\ Phys. Rev. 114, 1571 (1959). \\ ${ }^{19}$ D. J. Hughes, J. A. Harvey, M. D. Goldberg, and \\ M. J. Stafne, Phys. Rev. 90, 497 (1953). \\ ${ }^{20}$ V. E. Krohn, private communication. \\ ${ }^{21}$ S. F. Mughabghab and D. J. Garber, BNL Report \\ No. BNL 325, 1973 (unpublished), 3rd ed., Vol. 1.
}

\title{
Internal Pair Formation Following Coulomb Excitation of Heavy Nuclei*
}

\author{
Volker Oberacker, Gerhard Soff, and Walter Greiner \\ Institut für Theoretische Physik der Johann Wolfgang Goethe Universität, Frankfurt am Main, Germany
} (Received 26 January 1976)

\begin{abstract}
Internal conversion of $\gamma$ rays from Coulomb-excited nuclear levels cannot be neglected compared with the spontaneous and induced positron production in overcritical electric fields. It is shown that both processes are separable by their different distributions with respect to the ion angle and the positron energy.
\end{abstract}

In overcritical electric fields spontaneous positron production may occur as a result of an unoccupied electronic $1 s_{1 / 2}$ level entering the negative-energy continuum, ${ }^{1-4}$ which is equivalent to the decay of the neutral vacuum into a charged one. It has been proposed ${ }^{4}$ that the positron emission can be experimentally observed in collisions of very heavy ions with $Z_{1}+Z_{2}>Z_{\mathrm{cr}} \sim 170$. Because of the nonadiabaticity of the collision process $e^{+} e^{-}$pairs are additionally created by transitions from the negative-energy continuum to the $K$ shell; this "induced decay" increases the cross section by two orders of magnitude. ${ }^{3}$

However, there are several background effects leading to pair formation in heavy-ion scattering that have to be investigated carefully. Among these are conversion of timelike nuclear bremsstrahlung photons, direct pair production by the alternating Coulomb field, and internal conversion of $\gamma$ rays from nuclear transitions. It was shown by Reinhardt, Soff, and Greiner ${ }^{5}$ that the first process can be neglected $\left(\sigma_{\mathrm{brems}} e^{+} e^{-} \sim 10^{-8} \mathrm{~b}\right)$ while the second one is expected to be important only for relativistic ion velocities, since there is an exchange of two spacelike photons involved. The dominant background results from the decay of Coulomb-excited nuclear levels. Although the mean lifetime of nuclear quadrupole transitions with $\Delta E>2 m_{e} c^{2} \sim 1 \mathrm{MeV}\left(\tau_{\mathrm{dec}} \sim 10^{-13} \mathrm{sec}\right)$ is much larger than the collision time $\left(\tau_{\text {coll } 1} \sim 10^{-20} \mathrm{sec}\right)$ in which the spontaneous and induced positrons are emitted, an experimental separation of both processes seems not to be feasible. If the scattered nucleus and the positrons are measured in coincidence, the differential pair-creation cross section is given by the product of the scattering cross section and the positron emission probability of both nuclei:

$$
\begin{aligned}
& \frac{d^{2} \sigma_{\mathrm{Cb}} e^{+} e^{-}}{d E_{p} d \Omega_{\text {ion }}} \\
& \quad=\frac{d \sigma_{\text {scat }}}{d \Omega_{\text {ion }}}\left(\vartheta_{\text {ion }}\right) \sum_{1,2} \frac{d W_{\mathrm{Cb}} e^{+} e^{-}}{d E_{p}}\left(E_{p}, \vartheta_{\text {ion }}\right) .
\end{aligned}
$$

The probability $d W / d E_{p}$ is a function of the positron kinetic energy $E_{p}$ and the scattering angle $\vartheta_{\text {ion }}$. It depends on the Coulomb excitation probability $P^{\mathrm{Cb}}$ of the initial nuclear level, the branching ratio $P^{\gamma}$ for a photon transition into the final state, and the corresponding differential pairformation coefficient $d \beta / d E_{p}$ :

$$
\begin{aligned}
& \frac{d W_{\mathrm{Cb}}^{e^{+} e^{-}}}{d E_{p}}\left(E_{p}, \vartheta_{\text {ion }}\right) \\
& \quad=\sum_{i, f} P_{i}{ }^{\mathrm{Cb}}\left(\vartheta_{\text {ion }}\right) P_{i f}^{\gamma} \frac{d \beta_{i f}}{d E_{p}}\left(E_{p}\right) .
\end{aligned}
$$


For the vacuum-decay experiment kinetic ion energies just below the Coulomb barrier are required in order to get a deep "diving in" of the $K$ shell $\left(R \ll R_{\mathrm{cr}}=35 \mathrm{fm}\right.$ for $\left.Z_{1}+Z_{2}=184\right)$. In this energy region the Coulomb excitation is strongly influenced by the nuclear forces, causing destructive-interference effects in the angular distributions and excitation functions. Both excitation modes (Coulomb and nuclear) are contained in the collective-coupling potential of Oberacker, Holm, and Scheid. ${ }^{6}$ Since we are interested in the scattering of deformed even-even nuclei $\left({ }^{238} U-{ }^{238} U\right)$, the collective rotation-vibration model (RVM) ${ }^{7}$ has been applied to describe the intrinsic Hamiltonian. Only rotations and quadrupole vibrations are considered since these are the dominant degrees of freedom. The large Sommerfeld parameter $\eta$ (for U- U we have $\eta \sim 600$ even at the Coulomb barrier) allows a semiclassical treatment of the excitation mechanism. The results of two different types of calculations have been compared: In method 1 all states of the RVM below the fission barrier were taken into account. Excitations into the continuum or equivalently Coulomb-fission probabilities have been evaluated according to Holm and Greiner. ${ }^{8}$ Within a cou- pled-channel code the excitation amplitudes of 520 states are calculated. For computational reasons we considered only magnetic substates $M=0$ which is exact for $\vartheta_{\text {ion }}=180^{\circ}$ 。 In method 2 the ground-state band and the first $\beta$ - and $\gamma$-vibrational bands of the RVM were employed up to spin $40^{+}$, including, however, all contributing magnetic substates。

The branching ratios $P^{\gamma}$ for the various nuclear transitions [see Eq. (2)] follow from the $B(E 2)$ values predicted by the RVM; these already enter in the excitation process. The internal-pair-formation coefficients, which give the number of $e^{+} e^{-}$ pairs compared with outcoming photons, have been evaluated in the plane-wave Born approximation according to Rose ${ }^{9}$ and to Akhiezer and Berestetsky. ${ }^{9}$ The total conversion coefficients agree within $20 \%$ with the exact values using Coulomb wave functions for the electronic continuum state ${ }^{10}$ even for high charge numbers, whereas a wrong distribution with respect to the positron energy is obtained. Therefore, like in usual $\beta$ decay we have multiplied $d \beta / d E_{p}$ with the wellknown correction factor ${ }^{10}$ reflecting the different distributions of electrons and positrons at the nucleus and normalized this to the total value a)

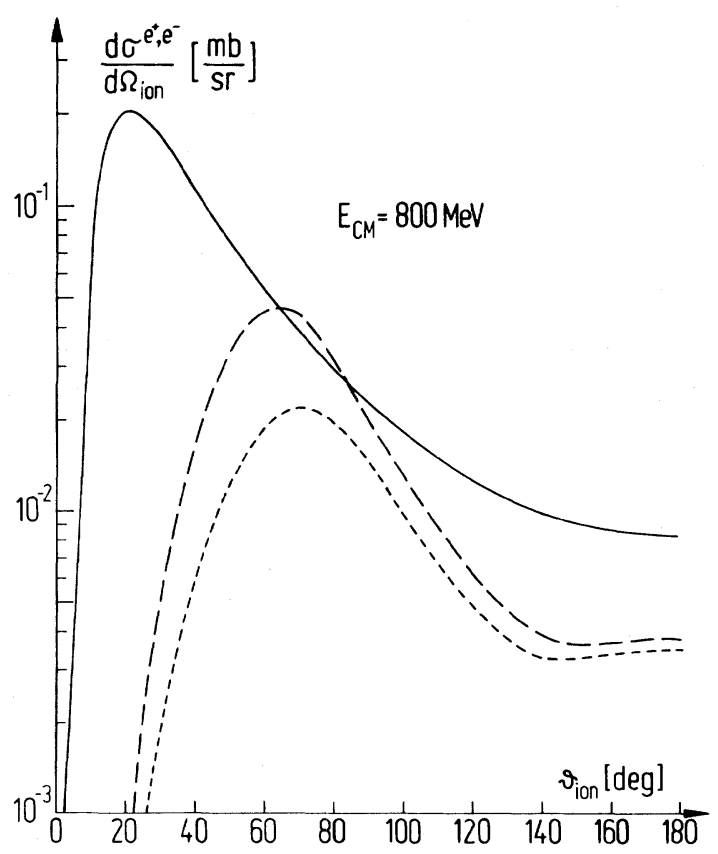

b)

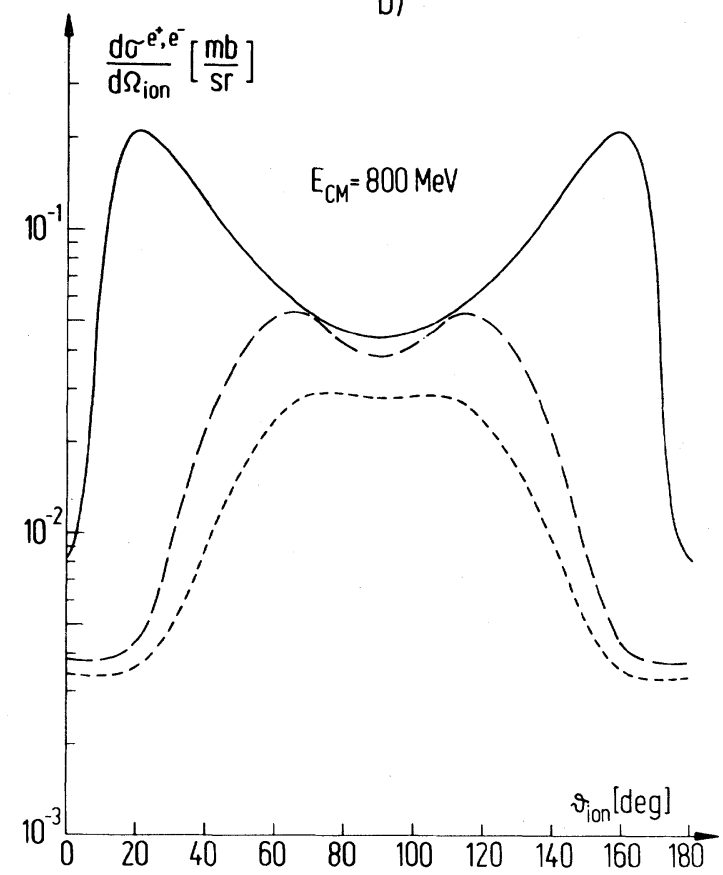

FIG. 1. The differential pair-formation cross sections (c.m.) with respect to the ion angle for (a) a nonsymmetric system (e.g., $\left.{ }^{234} \mathrm{U}-{ }^{238} \mathrm{U}\right)$ and (b) a symmetric system $\left({ }^{238} \mathrm{U}-{ }^{238} \mathrm{U}\right)$. The solid lines indicate the cross sections for $e^{+} e^{-}$pairs due to the spontaneous and induced decay of the vacuum $\left(L_{0}=10^{-2}\right.$ assumed). The dashed lines give the background from Coulomb and nuclear excitation calculated with method 1 (lower curve) and method 2 (upper curve), described in the text. 
from the plane-wave Born approximation. The numerical results for $e^{+} e^{-}$pair formation are shown in Fig. 1 for the U-U system at the Coulomb barrier. Figure 1(a) demonstrates the nonsymmetric cross sections with respect to the ion angle which will be measured in coincidence experiments allowing distinction between projectile and target nucleus (e.go, ${ }^{234} \mathrm{U}^{238} \mathrm{U}$ )。 In Fig。 1(b) the corresponding symmetric results are plotted for ${ }^{238} \mathrm{U}-{ }^{238} \mathrm{U}$ collisions. In both cases the dashed curves are for positrons due to Coulomb and nuclear excitation (calculated with methods 1 and 2 described above) while the solid lines are for those caused by the induced and spontaneous decay of the vacuum. ${ }^{11}$ Obviously, at rather forward angles the vacuum-decay positrons outnumber those from the nuclear background by several orders of magnitude. At $\vartheta_{\text {ion }}=20^{\circ}$, for instance, the ratio $R=d \sigma_{\mathrm{vac}} e^{+} e^{-} / d \sigma_{\mathrm{Cb}} e^{+e^{-}}$was found to be $R$ $\sim 300$ in the nonsymmetric distribution and $R \sim 50$ in the symmetric case. For backward ion angles, however, the cross sections only differ by a factor of 2. In the calculation of the spontaneous and induced positron production the $K$-vacancy probability $L_{0}$ was assumed to be $L_{0}=10^{-2}$. The corre-

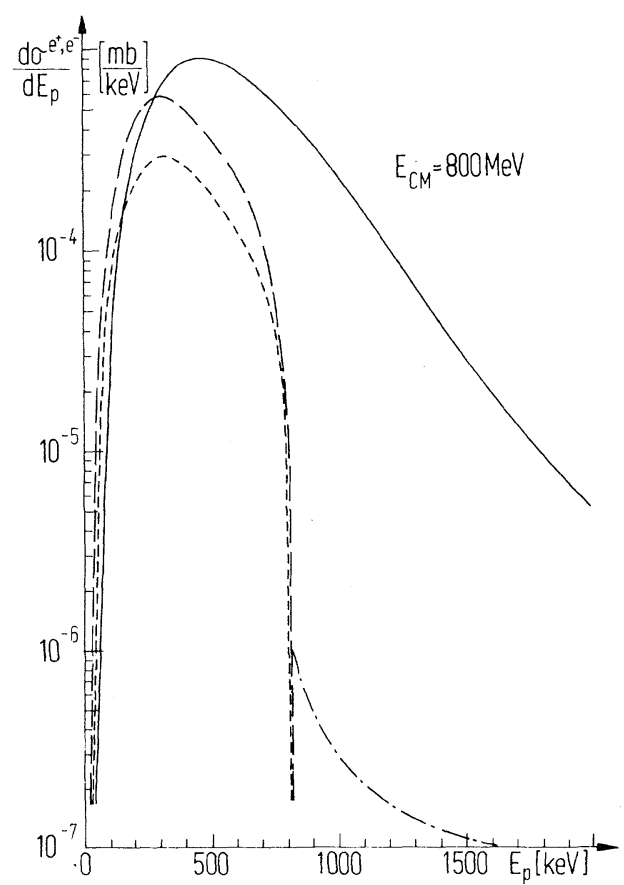

FIG. 2. Pair-creation cross sections as a function of the positron kinetic energy. The notation of the solid and dashed lines is the same as in Fig. 1. The dashdotted curve gives a semiquantitative estimate for the pair production due to higher band transitions in the ${ }^{238} \mathrm{U}$ nucleus. sponding total pair-formation cross sections are $\sigma_{\mathrm{vac}} e^{+} e^{-}=5.0 \times 10^{-4} \mathrm{~b}$ and $\sigma_{\mathrm{Cb}} e^{+} e^{-}=1.25 \times 10^{-4} \mathrm{~b}$ (method 1$)$ and $2.28 \times 10^{-4} \mathrm{~b}(\operatorname{method} 1)$.

The differential cross section with respect to the positron kinetic energy $E_{p}$ is obtained by integration of Eq. (1) over the solid angle $\Omega_{\text {ion }}$ 。 The results are shown in Fig. 2. Again, a characteristic difference is found between the pairs originating from Coulomb and nuclear excitation (dashed curves) and those from vacuum decay (solid curve). Since the maximum nuclear E2transition energy in ${ }^{238} \mathrm{U}$ is about $1.8 \mathrm{MeV}$, the positron spectrum terminates at $E_{p} \sim 800 \mathrm{keV}$ while the induced-positron spectrum contains much higher energies. Band mixing due to the rotation-vibration interaction has not been considered up to now. It allows higher nuclear transition energies which can be neglected, however, because of the small transition probabilities. For example, concerning the $\Delta E \sim 3-\mathrm{MeV}$ transition between the $2^{+}$member of the two-phonon vibrational band $\left(K=0, n_{2}=1, n_{0}=1\right)$ and the ground state we obtained $d \sigma_{\mathrm{Cb}} e^{+e^{-}} / d E_{p} \sim 10^{-9} \mathrm{mb} / \mathrm{keV}$ for $E_{p}>1 \mathrm{MeV}$. Even for 100 equivalent transitions the nuclear background still remains 2 or 3 orders of magnitude smaller than the vacuum-decay process, as indicated by the dash-dotted line in Fig. 2. This different behavior for positron kinetic energies $E_{p}>1 \mathrm{MeV}$ may also be used to distinguish experimentally between both processes. The uncertainties in the present calculations mainly arise from the very-high-spin states, where the RVM only yields approximate solutions, and from an influence of the octupole bands populated in Coulomb excitation of ${ }^{238} \mathrm{U}^{12}$ Furthermore, single-particle $E 1$ transitions with high energy cannot be excluded in principle. Since these effects are not able to change the order of magnitude, we can conclude that the observation of the positron emission in overcritical electric fields should be possible provided that the $K$-vacancy probability $L_{0}$ is larger than about $10^{-4}$.

In this connection it is important to realize that $K$-electron conversion can be induced by nuclearCoulomb excitation. One merely has to replace $\beta$ in Eq. (2) by the internal conversion coefficient for $K$-shell electrons. ${ }^{13}$ It results in a total production cross section of $\sigma_{K}{ }^{e-}=2.53 \mathrm{~b}$ for the U- U system at the Coulomb barrier. The corresponding $K$-vacancy probability as a function of the ion angle, $W_{K}{ }^{e-}\left(\vartheta_{\text {ion }}\right)$, is presented in Fig. 3. In contrast to the $K$-hole production induced by quasimolecular effects these $K$ holes are created after the collision $\left(\tau_{K} \sim 10^{-13} \mathrm{sec}\right)$. As a consequence, 


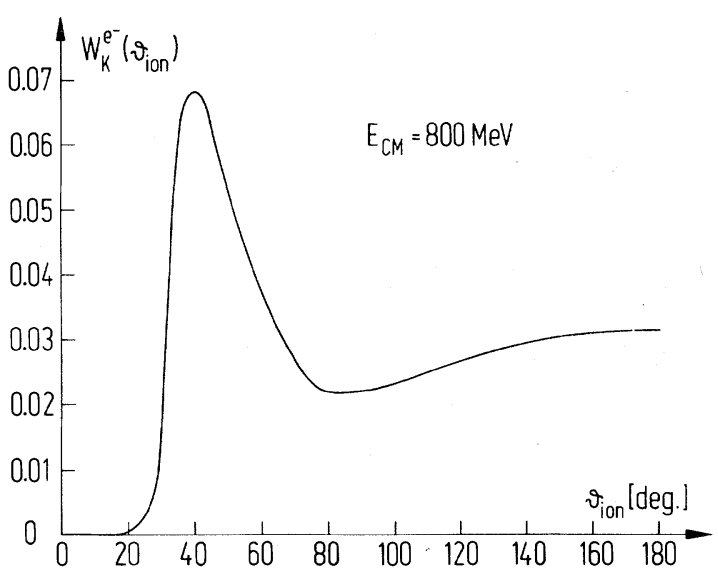

FIG. 3. $K$-hole production probability due to internal conversion induced by nuclear-Coulomb excitation with respect to the heavy-ion scattering angle in a ${ }^{234} \mathrm{U}-{ }^{238} \mathrm{U}$ collision.

the quasimolecular radiation should not be normalized to $K_{\alpha}$ transitions of separated atoms as done usually in experiments.

Finally it should be mentioned that spectroscopy of the $\mathrm{x}$ rays in superheavy quasimolecules is prevented in most cases for ion energies comparable to the Coulomb barrier. Our calculations concerning the U-U system indicate that the cross sections for nuclear photon transitions following Coulomb excitation and for molecular $K_{\alpha}$ transitions $\left(L_{0}=10^{-2}\right)$ are of the same order of magnitude in the energy region $E_{\gamma} \sim 600 \mathrm{keV}$, whereas at $E_{\gamma} \sim 900 \mathrm{keV}$ the nuclear contribution is already enhanced by roughly a factor 100 . At the unitedatom limit $\left(\Delta E_{2 p_{3 / 2}-1 s_{1 / 2}} \sim 1330 \mathrm{keV}\right.$ for $Z_{1}+Z_{2}$ $=184)^{14}$ the nuclear photon spectrum is absolutely dominant even for $L_{0} \sim 1$.

*This work has been supported by the Bundesministerium fur Forschung und Technologie, by the Gesellschaft für Schwerionenforschung, and by the Deutsche Forschungsgemeinschaft.

${ }^{1}$ W. Pieper and W. Greiner, Z. Phys. 218, 327 (1969).

${ }^{2}$ V. S. Popov, Zh. Eksp. Teor. Fiz. 59, 965 (1970)

[Sov. Phys. JETP 32, 526 (1971)].

${ }^{3}$ K. Smith, H. Peitz, B. Mưller, and W. Greiner, Phys. Rev. Lett. $\underline{32}, 554$ (1974).

${ }^{4} \mathrm{~J}$. Rafelski, L. P. Fulcher, and W. Greiner, Phys. Rev. Lett. 27, 958 (1971); B. Muller, H. Peitz, J. Rafelski, and W. Greiner, Phys. Rev. Lett. 28, 1235 (1972).

${ }^{5} \mathrm{~J}$. Reinhardt, G. Soff, and W. Greiner, to be published.

${ }^{6}$ V. Oberacker, H. Holm, and W. Scheid, Phys. Rev. C 10,1917 (1974).

${ }^{7} \mathrm{~J}$. M. Eisenberg and W. Greiner, Nuclear Theory (North-Holland, Amsterdam, 1970), Vol. 1.

${ }^{8}$ H. Holm and W. Greiner, Nucl. Phys. A195, 333 (1972).

${ }^{9}$ E. M. Rose, Phys. Rev. $\underline{76}, 678$ (1949); see also A. I. Akhiezer and V. B. Berestetsky, Quantum Electrodynamics, translated by Consultants Bureau, Inc. (Office of Technical Services, U. S. Department of Commerce, Washington, D. C., n.d.).

${ }^{10} \mathrm{R}$. Wilson, in Alpha -, Beta-and Gamma-Ray Spectroscopy, edited by K. Siegbahn (North-Holland, Amsterdam, 1965), p. 1557.

${ }^{11} \mathrm{H}$. Peitz, private communication.

${ }^{12}$ E. Grosse, J. de Boer, R. M. Diamond, F. S. Stephens, and P. Tjфm, Phys. Rev. Lett. 35, 565 (1975).

${ }^{13}$ R. S. Hager and E. C. Seltzer, Nucl. Data, Sect. A 14, 1 (1968).

${ }^{14}$ B. Mưller, R. K. Smith, and W. Greiner, Phys. Lett. 53B, 401 (1975). 